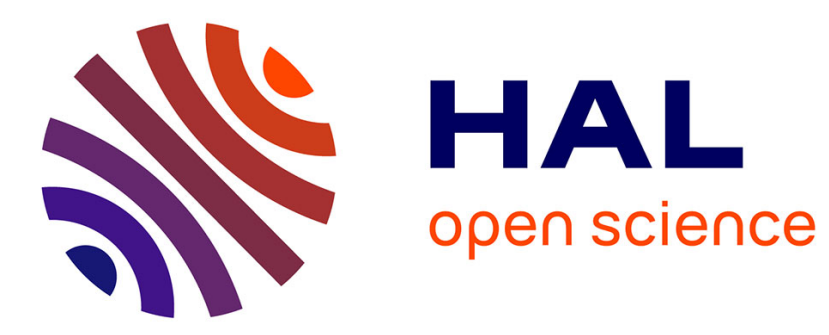

\title{
The dicrotic notch analyzed by a numerical model
}

\author{
María Teresa Politi, Arthur R. Ghigo, Juan Fernández, Ismaïl Khelifa, Julien
}

Gaudric, J.-M. Fullana, Pierre-Yves Lagrée

\section{To cite this version:}

María Teresa Politi, Arthur R. Ghigo, Juan Fernández, Ismaïl Khelifa, Julien Gaudric, et al.. The dicrotic notch analyzed by a numerical model. Computers in Biology and Medicine, 2016, 72, pp.54-64. 10.1016/j.compbiomed.2016.03.005 . hal-01288428

\section{HAL Id: hal-01288428 \\ https://hal.sorbonne-universite.fr/hal-01288428}

Submitted on 15 Mar 2016

HAL is a multi-disciplinary open access archive for the deposit and dissemination of scientific research documents, whether they are published or not. The documents may come from teaching and research institutions in France or abroad, or from public or private research centers.
L'archive ouverte pluridisciplinaire HAL, est destinée au dépôt et à la diffusion de documents scientifiques de niveau recherche, publiés ou non, émanant des établissements d'enseignement et de recherche français ou étrangers, des laboratoires publics ou privés. 
Full title: The dicrotic notch analyzed by a numerical model.

Abbreviated title: The dicrotic notch analyzed by a numerical model.

Authors:

Politi, María Teresa ${ }^{3 *}$

Ghigo, Arthur ${ }^{1 *}$

Fernández, Juan Manuel ${ }^{3}$

Khelifa, Ismaï ${ }^{2}$

Gaudric, Julien ${ }^{1,2}$

Fullana, José María ${ }^{1}$

Lagrée, Pierre-Yves ${ }^{1}$

1: Sorbonne Universités, UPMC Univ Paris 06, CNRS, UMR 7190, Institut Jean Le Rond d'Alembert, F-75005 Paris, France

2: Service de Chirurgie Vasculaire, Hôpitaux Universitaires La Pitié-Salpêtrière, 47-83 Bd de l'Hôpital 75013 Paris, France.

3: Laboratorio de Biomembranas. Instituto de Fisiología y Biofísica Bernardo Houssay (IFIBIO Houssay). Facultad de Medicina, Universidad de Buenos Aires. Paraguay 2155, C1121ABG Buenos Aires, Argentina.

* Both authors contributed equally to this study.

Corresponding author:

Arthur R. Ghigo

arthur.ghigo@,dalembert.upmc.fr

Institut Jean Le Rond $\partial$ 'Alembert

Université Pierre et Marie Curie

4 Place Jussieu, 75005, Paris, France

Phone: (+33) 0144273790

María Teresa Politi

teresapoliti@yahoo.com.ar

Laboratorio de Biomembranas

Instituto de Fisiología y Biofísica Bernardo Houssay (IFIBIO Houssay).

Facultad de Medicina, Universidad de Buenos Aires.

Ciudad Autónoma de Buenos Aires, C1121ABG.

Phone and fax: (+5411) 5950-9500 (intern 2145) 


\section{The dicrotic notch analyzed by a numerical model.}

\section{Abstract}

Divergent concepts on the origin of the dicrotic notch are widespread in medical literature and education. Since most medical textbooks explain the origin of the dicrotic notch as caused by the aortic valve closure itself, this is commonly transmitted in medical physiology courses. We present clinical data and numerical simulations to demonstrate that reflected pressure waves could participate as one of the causes of the dicrotic notch. Our experimental data from continuous arterial pressure measurements from adult patients undergoing vascular surgery suggest that isolated changes in peripheral vascular resistance using an intravenous bolus of phenylephrine (a selective alpha 1-receptor agonist and thus a potent vasoconstrictor) modify the dicrotic notch. We then explore the mechanisms behind this phenomenon by using a numerical model based on integrated axisymmetric Navier-Stokes equations to compute the hemodynamic flow. Our model illustrates clearly how modifications in peripheral artery resistance may result in changes in the amplitude of the dicrotic notch by modifying reflected pressure waves. We believe that this could be a useful tool in teaching medical physiology courses.

\section{Highlights}

- Aortic valve closure is the most widespread explanation for the dicrotic notch.

- Reflected pressure waves may participate in forming the dicrotic notch.

- A 1D numerical model based on Navier-Stokes equations can test this hypotheses.

- Changes in terminal resistance modify reflected waves and thus the dicrotic notch.

- Vasoconstrictors modify the morphology of the dicrotic notch in human subjects.

Keywords

Dicrotic notch. Reflection wave. Numerical model. Medical physiology.

\section{Introduction}

The dicrotic notch is a small and brief increase in arterial blood pressure that appears when the aortic valve closes. This landmark has been widely referred to in the descriptive analysis of the arterial waveform (especially of aortic and radial arteries) and is commonly used as an equivalent of end-systolic left ventricular pressure $(8,11)$.

Most medical textbooks explain the origin of the dicrotic notch as caused by the aortic valve closure itself $(5,10,19,26,29,34)$. These authors claim that towards the end of the ejection phase, blood flow across the aortic valve falls to very low values, until it actually reverses, producing a backflow that closes the aortic valve. As both flow and pressure are strongly coupled, this momentary backflow would produce a small positive shift in the aortic pressure trace. The dicrotic notch would therefore be the result of a short period of backward flow of blood immediately before the aortic valve closes. Many studies support this theory and have stressed that both events occur simultaneously $(14,15)$.

On the other hand, there are clinical data that emphasize that the morphology of the dicrotic notch is related to the value of mean arterial pressure and, therefore, to peripheral vascular resistance $(7,11)$. Peripheral vascular resistance is mainly influenced by the vascular tone of small distal arteries (diameter smaller than $500 \mu \mathrm{m}$ ), called 
arterioles (18). Pressure waves created by the heart travel through the arteries and reflect back towards the heart at places with high-vascular tone (such as the arterioles) and also at every vascular bifurcation (20). Hence, blood pressure waveforms can be viewed as a large addition of the forward pressure waves generated by the heart and the backward components due to multiple reflections, in particular, from the sites of distal resistance $(17,24,33)$. This backward component would modify the mean arterial pressure and also change the morphology of the forward pressure wave, resulting in the creation of the dicrotic notch. The speed of these reflected waves depends on the mechanical and geometrical characteristics of the vessels (25) but their amplitude depends directly on the value of peripheral resistance, that is, on the degree of vasoconstriction or vasodilation of the arterioles $(30,32)$. In the following, the influence of the peripheral resistance will be quantified by a reflection coefficient $(\mathrm{Rt}=\mathrm{Ra} / \mathrm{Ia})$, which is the ratio of the reflected amplitude ( $\mathrm{Ra}$ ) to the incident amplitude (Ia). Some authors state that a high vascular tone also increases the speed of the reflected waves (34). We consider that this would only change the amplitude of the reflected waves, not the speed, and will be addressing this matter in our experiments.

Based on this hypothesis, the presence of the dicrotic notch, created by the reflected waves, would induce a secondary pressure peak in the aorta, interrupting the aortic pressure downslope during end-systole. The increased pressure gradient between the aorta and the left ventricle would be sufficient to create a momentary backflow and close the aortic valve at higher diastolic levels, explaining why both the dicrotic notch and the aortic valve closure occur at the same time. We have arguments to put forward that this second approach responds to a better understanding of how the cardiovascular system actually works.

Understanding the dicrotic notch as a result of reflected pressure waves suggests that this landmark contains valuable information on the peripheral vascular network. We believe that this is an important concept to underline in cardiovascular physiology courses; therefore we present a teaching example that combines theoretical reasoning, numerical modeling and human experimental data. We propose a 1D model to study the role of reflected waves in the origin of the dicrotic notch, and compare the results from numerical simulations to experimental data from adult patients.

\section{Methods}

\section{Invasive arterial pressure measurements in representative patients}

Experimental data were obtained from continuous invasive arterial pressure measurements with a fluid-filled catheter from the right radial artery of adult patients undergoing peripheral vascular surgery at the Hôpital Universitaire Pitié-Salpêtrière in Paris, France. The study was approved by the IRB of the Hopitaux Universitaires La Pitié-Salpetrière, adheres to the requirements of the U.S. Federal Policy for the Protection of Human Subjects (45 CFR, Part 46), and is in accordance with the ethical principles of the Declaration of Helsinki. We used a disposable pressure transducer (TruWave, Edwards Lifesciences ${ }^{\circledR}$ ) with a natural frequency of $40 \mathrm{~Hz}$ for a standard kit, which has shown to be appropriate for measuring blood pressure $(9,28)$. Data were registered using an analogue-digital converter (MP150, BIOPAC Systems Inc.) and the AcqKnowledge software. Data acquisition rate was $100 \mathrm{~Hz}$.

Quantitative waveform analysis was done by measuring the time between the minimum value of the original radial pressure wave and the peak value of the reflection wave. 
Additionally, the relative time-position of the reflected wave within the pressure cycle (i.e., time between the minimum value of the original pressure wave and the peak value of the reflection wave divided by the overall cycle length) was studied to test the hypothesis of the reflection wave travelling faster as a result of the increased vascular tone. Systolic and diastolic arterial pressure values were computed from the maximum and minimum values of the original radial pressure wave. The amplitude of the dicrotic notch was computed from the peak value of the reflection wave. After defining a timewindow for each of these points by visual assessment, exact locations were identified using the first time-derivative of the pressure signal $(\mathrm{dP} / \mathrm{dt})$. Values were averaged over a 10-second interval. We compared data from before and after an intravenous bolus of phenylephrine $(50-100 \mu \mathrm{g})$, which is a selective alpha 1-receptor agonist and thus a potent vasoconstrictor without any direct effect on heart rate and contractility. A brief transitional period was allowed between one state and the other. To better compare the dicrotic notch before and after the phenylephrine IV bolus, time was normalized to the duration of one cycle and pressure was normalized to the nearest inflexion point before the dicrotic notch (identified as the local minimum of the first time-derivative). Statistical analysis involved paired-Student's t-test (two-tailed) for before-after testing and independent Student's t-test (two-tailed) for comparisons among groups $(\alpha=0.05)$ using R studio free statistical software. Data are presented as mean \pm standard deviation.

\section{Theoretical reasoning and numerical model}

The aim of the numerical model is to accurately describe the flow of blood in the systemic network. Blood is a non-Newtonian fluid, governed by the 3D Navier-Stokes equations, interacting with the viscoelastic arterial wall as it propagates through the network. The computational cost of solving this problem numerically even for a single vessel is extremely high. Through the following series of relevant approximations, the complexity of the problem can be reduced. A simplified model of the systemic network is considered, using only a limited number of arteries. The heart is modeled by a periodic flow input signal (Figure 3), and therefore no retroaction between the flow in the aorta and the heart is considered. The influence of the peripheral vascular bed is taken into account through a terminal reflection coefficient (Rt) set individually for each terminal segment. The geometry of the arteries is simplified to straight tubes with a circular cross-section and viscoelastic walls, in which the shear rate is high enough to consider blood as a Newtonian fluid. Combining the previous hypotheses, the NavierStokes equations can be simplified to the following 1D equations, expressed in terms of the cross-section area (A) and the flow rate (Q), describing the flow of blood and its interaction with the viscoelastic arterial wall in each artery of the model for all times:

$\partial_{t} A+\partial_{x} Q=0$

Equation 1.

$\partial_{t} Q+\partial_{x}\left(\frac{Q^{2}}{A}+\frac{\beta}{3 \rho} A^{\frac{a}{2}}\right)=-C_{f} \frac{Q}{A}+C_{v} \partial_{x x} Q$

Equation 2.

where $\rho$ is the density of the fluid and $C_{f}$ is the viscous friction coefficient. The coefficients

$$
\beta=\frac{\sqrt{\pi} E h}{\left(1-v^{2}\right) A_{0}}
$$

and 


$$
C_{V}=\frac{\sqrt{\pi} h \phi}{2 \rho\left(1-v^{2}\right) \sqrt{A_{0}}}
$$

respectively represent the elastic (or compliant) and viscoelastic response of the arterial wall and contain the physical parameters of the problem. We used the Young's modulus $E$, the Poisson ratio $v$, the viscoelastic coefficient $\phi$, the neutral cross-sectional area $A_{0}$ and the arterial thickness $h$. These parameters are prescribed for each artery of our model network (31). More details on the assumptions of this model can be found in Ambrosi et al and in Caro et al $(3,6)$.

Despite these simplifications, this system of equations contains all the necessary physical behaviors to accurately describe blood flow in a network. The most important feature is its ability to capture the propagation of waves along the arteries, as a result of the elastic properties (or compliance) of the arterial wall, represented by the coefficient $\beta$ in equation 2. As these waves propagate in the network, the viscosity of the wall (described by the coefficient $\mathrm{C}_{v}$ in equation 2) and the viscosity of the fluid (described by the coefficient $\mathrm{C}_{\mathrm{f}}$ in equation 2 ) are responsible respectively for the diffusion of the signal in the axial direction and its attenuation. The model also describes the reflection of the waves at each bifurcation and terminal segment. The coefficient $\mathrm{Rb}$ represents the reflection occurring at a bifurcation and depends on the geometrical and mechanical properties of the parent and daughter arteries of the bifurcation. On the contrary, terminal reflection coefficients (Rt) are imposed numerically. These terminal reflection coefficients are set to model the reflective (or resistive) behavior of the peripheral vascular network. It is important to note that both internal and terminal reflections play an important role in modifying the waveforms as they propagate in the network.

In the following numerical computations, we used three different simplified models of the systemic network (Figure 1). The first was taken from the literature and represents the 55 principal segments of the human arterial network, which is a simplified construction based on vascular nuclear magnetic resonance images. The geometrical and mechanical parameters for each of the 55 segments were taken from the literature (31). For the sake of simplicity, the reflection coefficients for all terminal segments had the same value, Rt. The second model describes a single artery. The mechanical and geometrical parameters of this single segment are identical to those of the aorta of the 55 arteries model and can be found in Table 1.The third model is a single bifurcation formed of one parent artery and two daughter arteries. The geometrical and mechanical parameters of the parent artery are those of the single artery model. The two daughter arteries are identical and their parameters are presented in Table 2.

The governing equations are solved using a finite volume method that was validated on in vitro data (31).

\section{Results}

\section{EXPERIMENTAL DATA}

In order to test the hypothesis that the presence of the dicrotic notch is related to the degree of peripheral vasoconstriction, we recorded the arterial pressure waveform registered continuously from the arterial line of patients undergoing peripheral vascular surgery. Data were taken from short, stable periods during the surgery, in which the only modification introduced was the injection of an intravenous bolus of phenylephrine. No vascular clamping or additional drug modifications were taking 
place at the time. Periodic oscillations in pressure waves were due to respiratory variations, which may be emphasized in patients under mechanical ventilation.

Figure 2A shows data from Patient 1, an 81 year-old female smoker with arterial hypertension undergoing abdominal aorta prosthetic replacement after the diagnosis of an infrarenal abdominal aortic aneurysm. After an IV bolus of $100 \mu \mathrm{g}$ of phenylephrine, not only did the systolic arterial pressure increase significantly from 94.1/52.3 to $109.5 / 56.1 \mathrm{mmHg}(\mathrm{p}<0.01)$, but also the peak value of the dicrotic notch became higher $(56.2 \pm 2.9$ vs. $60.2 \pm 2.8 \mathrm{mmHg} ; \mathrm{p}<0.01)$ (Figure $2 \mathrm{~A}$; left and middle panels). To additionally compare the dicrotic notch before and after the phenylephrine IV bolus, time was normalized to the duration of one cycle and pressure was normalized to the nearest inflexion point before the dicrotic notch. There was a statistically significant increase in the normalized peak value of the main wave (1.28 \pm 0.04 vs. $1.31 \pm 0.03$; $p=0.03)$ and in the normalized peak value of the dicrotic notch $(0.71 \pm 0.01$ vs. $0.76 \pm 0.02 ; p<0.01)$ after the IV bolus of phenylephrine. The proportional increase in the normalized peak value of the main wave $(2 \%)$ was significantly smaller than the proportional increase in the normalized peak value of the dicrotic notch $(6 \%)(p<0.01)$ (Figure 2A; right panel). The time between the minimum value of the original pressure wave and the peak value of the dicrotic notch did not change after phenylephrine injection $(0.40 \pm 0.02$ vs. $0.41 \pm 0.03$ seconds; $p=0.35)$ nor did the relative time-position of the dicrotic notch within the pressure cycle $(0.69 \pm 0.03$ vs. $0.69 \pm 0.04 ; p=0.88)$. These results indicate that, after vasoconstriction, the dicrotic notch only increases its amplitude; it does not appear earlier in the cycle.

Figure 2B shows the arterial pressure waveform of Patient 2, a 56 year-old obese female without any known history of heart disease, undergoing a right femoral-popliteal bypass. Continuous invasive pressure measurements show that with an initial blood pressure of $124.5 / 82.4 \mathrm{mmHg}$ the patient had a monophasic waveform that lacked any noticeable dicrotic notch. After an IV bolus of $50 \mu \mathrm{g}$ of phenylephrine, blood pressure increased significantly up to $195.6 / 105.2 \mathrm{mmHg}(\mathrm{p}<0.01)$ and waveform morphology changed showing a clear dicrotic wave interrupting the end of the diastolic slope.

Since in both cases the only modification introduced between the two states was the addition of a powerful and selective vasoconstrictor, phenylephrine, the presence (or the increased amplitude) of the dicrotic notch could be attributed to an increase in the peripheral vascular tone. These experimental results suggest that the presence and the amplitude of the dicrotic notch are correlated with the value of peripheral resistance. Nevertheless, since we did not measure cardiac output or pulse wave velocity, we cannot assure there is a causal relationship between the two, based on our experimental data.

Given the complexity of a human experimental model, a theoretical model of the human vascular network is required to explore the relationship between the amplitude of the dicrotic wave and the value of peripheral resistance.

\section{NUMERICAL SIMULATIONS}

In order to explore the hidden mechanisms behind the relationship between the amplitude of the dicrotic notch and vascular tone, while overcoming the difficulties of obtaining invasive data from humans under different conditions, we propose a numerical model of the human systemic arterial tree. 
We first present the results obtained using the arterial network of 55 segments, then with the single segment model considering one straight artery without bifurcations, and finally with a single segment model with a bifurcation (one parent artery that divides into two daughter arteries).

\section{5-SEGMENT MODEL}

Numerical simulations compute the flow rate $(\mathrm{Q})$ and the pressure $(\mathrm{P})$ at every chosen record point of the 55-segment vascular network. The time evolution of these variables was studied in the proximal aorta, as to correlate with experimental data from other studies. To study the influence of the shape of the signal as well as backflow, three different inlet flow rate signals were considered in order: a parabolic signal without backflow (normal), a parabolic signal with backflow (backflow), and a triangular signal without backflow (triangular) (Figure 3). The amplitude of the different signals was set so that the total volume of blood ejected during each cycle is identical for each input signal considered. Backflow was simulated considering a maximum amplitude that is one fifth of overall aortic flow $(\mathrm{Qmax} / 5)$ and a period that is one sixth of overall aortic flow period (Tc/6), which probably overestimates real values. Results show that for each inlet signal considered, a terminal coefficient of $\mathrm{Rt}=0$ yields a monophasic pressure-time curve that perfectly mirrors flow-time curves and lacks a dicrotic notch. On the other hand, a terminal coefficient of $\mathrm{Rt}=0.4$ results in a clear dicrotic notch which interrupts the downward pressure slope. An even higher terminal coefficient of $\mathrm{Rt}=0.8$ produces a higher dicrotic notch that is overimposed on the first pressure wave.

These numerical results from entire network simulations suggest that 1) the presence and the amplitude of the dicrotic notch can be modified by simply changing the terminal reflection coefficient, and 2) the presence of the dicrotic notch does not depend on the shape of the inlet flow signal. Backflow in the aorta is not required to create a dicrotic notch. However, using different inlet signals will modify the shape of the dicrotic notch, since the reflected waves will not be the same.

These results are in good accordance with our hypothesis that inlet backflow does not create the dicrotic notch by itself. In the following simulations, we will therefore consider only parabolic inlet signals without backflow.

\section{SINGLE SEGMENT MODEL WITHOUT BIFURCATIONS}

In real experiments as well as in numerical simulations using the 55 arteries model, it is difficult to understand the underlying mechanism responsible for the creation of the dicrotic notch, since so many effects come into play. To simplify the problem, we used a very reduced model of the 55-segment arterial network, considering only one artery without bifurcations. In this situation, the variables of interest that influence wave morphology (such as terminal reflection (Rt) coupled with classical compliant $(\beta)$, viscous $\left(\mathrm{C}_{\mathrm{f}}\right)$ and viscoelastic $\left(\mathrm{C}_{\mathrm{v}}\right)$ effects) can be easily modified, identified and therefore studied. We sought to evaluate the influence of each of these variables on the dicrotic notch through numerical simulation.

We computed the pressure-time curve of a single-beat impulse in arteries of different lengths $(\mathrm{L}=1,000 \mathrm{~mm}, 600 \mathrm{~mm}, 400 \mathrm{~mm}$ and $200 \mathrm{~mm})$ and plotted these curves in the same figure. We chose this strategy because the reflection waves resulting from the original pressure wave can be clearly identified in single-beat simulations. Likewise, 
individual reflection waves can be easily pointed out in longer vessels, since the increased vessel length prolongs the travelling time between the original pressure wave and the reflection point, separating the waves apart. Regardless of the overall length of the artery, the signal was always measured in the same location (i.e., at $150 \mathrm{~mm}$ from the beginning of the artery). Since the heart signal is periodic and has two phases (systole and diastole) and ejection occurs only during systole, we considered that ejection time was half the pulse period by supposing that each phase occupies half of the entire pulse period and that the systolic phase is mainly occupied by the ejection period (which is only slightly different from physiologic findings).

\section{Pure wave propagation}

We considered first a pure wave propagation, suppressing all source of attenuation and diffusion (meaning that no viscous or viscoelastic effects were taken into account, that is, both coefficients $\mathrm{C}_{\mathrm{f}}$ and $\mathrm{C}_{v}=0$ ), with a pulse period of 0.1 seconds (i.e., ejection time $=0.05$ seconds). We initially considered a reflection coefficient (Rt) of 0 . As expected, our simulation showed a single original pressure wave, that was identical regardless of the vessel length, and no reflected waves (Figure 4A). When we increased the reflection coefficient up to $\mathrm{Rt}=0.4$ (Figure $4 \mathrm{~B}$ ), two additional reflected pressure waves appeared, which had identical amplitudes but were recorded at different times for each vessel length. These two waves are the result of the reflection of the original pressure wave at the end of the artery and the reflection of the first reflected wave at the beginning of the artery. In shorter vessels the two reflection waves are much closer to the original pressure wave than in longer vessels (e.g., compare the reflection waves from the $600 \mathrm{~mm}$ vessel and the $1,000 \mathrm{~mm}$ vessel). As the length of the artery decreases, the two reflection waves move closer and closer to the original pressure wave, since the first reflection wave is travelling a smaller distance at the same speed. Eventually, at very short vessel lengths, the two reflection waves merge together with the original wave by forming a notch (i.e., the $200 \mathrm{~mm}$ vessel). This clearly shows how the final morphology of the pressure-time waveform is the addition of the original pressure wave and several reflection waves. When we increased again the reflection coefficient up to $\mathrm{Rt}=0.8$ (Figure $4 \mathrm{C}$ ), the pressure-time waveform was similar to Figure 4B but had a higher amplitude. It is important to note that by increasing the reflection coefficient, only the amplitudes of the waves change. The reflected waves do not get closer to one another nor to the original pressure wave, that is, they do not increase their propagation velocity: the waves arrive at $150 \mathrm{~mm}$ at the same time, regardless of the reflection coefficient.

These numerical simulations show that the reflection waves originated at the terminal reflection site can create a notch on the original pressure and that the amplitude of these waves depends on the value of the terminal coefficient (Rt).

Up to this point, we have considered a pulse period of 0.1 seconds, since this allows us to identify individual waves more easily. Nevertheless, this would imply a heart rate of 600 beats per minute. A pulse period of 0.8 seconds would be a more realistic approach, yielding a heart rate of 75 beats per minute. Pressure-time curve simulations considering a pulse period of 0.8 seconds (i.e., ejection time $=0.4$ seconds) show that, once again, reflected waves disappear when the terminal reflection is zero (Figure 5A) and increase in amplitude as the terminal reflection does (Figures 5B and 5C). With a longer pulse period, the reflected waves merge with the original pressure wave even in vessels with 
long lengths (e.g., in the $600 \mathrm{~mm}$ vessel they are almost completely merged together) (Figure 5B) and the individual elements of these pressure waves are harder to point out.

Considering only the propagation of a wave without viscous attenuation or viscoelastic diffusion, we were able to show that in the scope of our experiments, it was possible to form a dicrotic notch through positive interactions between reflected waves originated at the terminal reflection site and the original pressure wave. Furthermore, we showed that when considering a realistic pulse period of 0.8 seconds, the interactions between the forward and backward travelling wave were strengthen.

\section{Viscous and viscoelastic effects}

The influence of viscous and viscoelastic effects (respectively governed by the coefficients $\mathrm{C}_{\mathrm{f}}$ and $\mathrm{C}_{\mathrm{v}}$ ) was added in the following series of simulations. Once again, we considered a pulse period of 0.1 seconds in order to compare with pure wave propagation curves. Figure $6 \mathrm{~A}$ shows that with a reflection coefficient (Rt) of 0 there is a single original pressure wave and no reflected waves. Figures $6 \mathrm{~B}$ and $6 \mathrm{C}$ represent numerical solutions for reflection coefficients (Rt) of 0.4 and 0.8 , respectively, and we observe reflected waves that have higher amplitude for higher reflection coefficient. In comparison to pure wave propagation, the reflected waves merge with the original pressure wave at longer lengths (e.g., in the $400 \mathrm{~mm}$ vessel) when influenced by viscous and viscoelastic effects.

These simulations show that the presence of viscosity and viscoelasticity "smoothens the curves out", resulting in the overlap of waves that previously were identified as individual elements when considering only pure wave propagation. These results show that, when considering viscous and viscoelastic effects, the overlap between waves is greater but still dependent on the reflection terminal coefficient.

\section{Changes in elasticity}

The effects of changing the vessel elasticity (E) were studied using the same single artery model as before, this time with a length of $600 \mathrm{~mm}$. Figure 7A indicates how, as the elasticity increases, the incoming pressure wave appears earlier (since the propagation speed is increased) and reaches higher values; however since the reflection coefficient (Rt) is 0 , the shape of the signal remains the same and does not form a dicrotic notch. Figures 7B and 7C consider a reflection coefficient (Rt) of 0.4 and 0.8 , respectively; thus reflected waves now appear and modify the shape of the signal, creating a dicrotic notch. In both these figures, vessels with a higher elasticity have higher and earlier reflected waves.

These results suggest that an increase in the vessel elasticity could change the overall morphology of the wave by increasing the propagation speed and therefore changing the timing of interaction between the incoming and the reflected waves. However, this depends on the creation of reflected waves, which are caused by terminal reflections and not changes in elasticity.

\section{SINGLE SEGMENT MODEL WITH A BIFURCATION}

As we mentioned previously, reflection can also occur at vessel bifurcations. We considered only one parent artery that divides into two daughter arteries. We studied the pressure-time curve of a single-beat impulse in parent arteries of different lengths $(1,000 \mathrm{~mm}, 600 \mathrm{~mm}, 400 \mathrm{~mm}$ and $200 \mathrm{~mm})$, keeping the geometrical and mechanical 
properties of the two daughter arteries constant. Furthermore, we set the reflection coefficients at the end of the daughter arteries to zero $(\mathrm{Rt}=0)$, ensuring that if a reflection occurred, it could only come from the bifurcation. The value of the reflection coefficient produced by the bifurcation depends on the geometrical and mechanical properties of the parent and the two daughter arteries, and in the configuration we consider its value is $\mathrm{Rb}=0.71$. Regardless of the overall length of the artery, the signal was always measured in the same location (i.e., at $150 \mathrm{~mm}$ from the beginning of the parent artery) and a pulse period of 0.1 seconds (i.e., ejection time $=0.05$ seconds) was used. As for the single-artery model without bifurcations, we considered only pure wave propagation (Figure 8). The results presented in Figure 8 are identical to those obtained for the single-artery model without bifurcations with a non-zero reflection coefficient (Figure 4B). Hence we demonstrated that internal reflection could also play an important role in the reflective behavior of the network and influence the shape of the dicrotic notch.

\section{Discussion}

Although the dicrotic notch withholds valuable information on cardiovascular hemodynamics, medical textbooks and literature explain its origin in very different ways, often leading to divergent interpretations among medical professionals.

We have shown through both numerical simulations and experimental data from adult patients that reflected waves may have a role in the origin of the dicrotic notch. Our results also suggest that increases in peripheral resistance increase the amplitude but not the propagation speed.

A commonly considered etiology of the dicrotic notch is that it is a manifestation of aortic valve closure $(5,10,19,26)$. However, this explanation could be incomplete. While the closure of the aortic valve generates a transient reversal of blood flow in the aorta which can contribute slightly to the shape of the notch (Figure 3), the only reasonable explanation for the aortic valve to close at all is the presence of a pressure gradient in that direction. This pressure gradient may be caused by the declining ventricular pressure during diastole and by the transient increase in aortic pressure caused by reflection waves, commonly referred to as the dicrotic notch. We therefore believe that even though the appearance of the dicrotic notch is simultaneous to the aortic valve closure, this explanation on its own may not be enough, and could be a lost chance for reinforcing important hemodynamic concepts.

Other authors do recognize the role of reflected waves in the origin of the dicrotic notch, but consider that a rise in the value of the reflection coefficient increases not only the amplitude of the reflection waves but also the speed at which they travel (34). Our experiments in patients undergoing vascular surgery show that after an IV bolus of phenylephrine the dicrotic notch either appeared if it was previously absent or it increased its amplitude if it was previously present (Figure 2). Nevertheless, in our experiments the relative time-position of the reflection wave in the overall pressure wave cycle remained unchanged. Although our experimental data have some limitations, since continuous pressure was not recorded using a high-fidelity transducer (only a fluid-filled system) and did not asses other variables in the cardiovascular system (such as cardiac output, pulse wave velocity or vasoactive responses), these 
findings are in accordance with our numerical simulations and with the concept that the speed at which pressure waves travel in a vascular network depends only on the structural characteristics of the network. For example, in elderly patients with vascular stiffening, reflected waves may move faster and reach the original arterial pressure waveform before it has time to decrease, which causes an amplification of the systolic pressure and, as a consequence, isolated systolic hypertension. This clinical condition illustrates clearly how changes in vascular elasticity can have an influence on the timeposition and the amplitude of the dicrotic notch. Indeed, several studies have used the dicrotic notch as a time-reference point to measure pulse wave velocity and as an indicator of arterial stiffness $(12,13)$. However, our results suggest that this effect would be greatly influenced by modifications in reflected waves, as shown in Figure 7, where, in the absence of reflected waves, changes in elasticity do not generate a dicrotic notch.

This problem was approached using a 1D model, which has been previously used to study the effects of reflected waves on vascular networks both numerically $(1,27)$ and experimentally $(16,4)$. Alastruey et al. used this method to conclude that the dicrotic notch is a combination of several mechanisms -mainly left ventricle outflow, arterial junction reflections and aortic valve closure. However these results were obtained through a linear model that did not include viscoelasticity, complementary experimental data, or a systematical study of the dicrotic notch (2). Mynard et al. also studied arterial wave reflections using a 1D model, this time introducing a heart model (23). However these authors did not systematically study the dicrotic notch either. These previous studies support the accuracy of this modeling approach for wave reflection analysis. The main contribution of our research is the systematical study of the role of reflected waves in the origin of the dicrotic notch, starting from experimental data from adult patients, and then complementing our analysis with data from a 1D numerical model -first with a 55 artery model and then with a single artery, progressively making the system simpler.

There are some limitations in the 1D numerical model. First, it does not include a heart model, so it does not take into account any feedback between the heart and the vascular network. A possible solution for this problem could be the heart model proposed by Mynard et al (23). Second, the arterioles, capillaries and veins are all simulated by the terminal reflection coefficient (Rt). A more accurate alternative would be to use a Windkessel model to estimate systemic resistance and characteristic impedance, with the additional costs in complexity.

Both our experimental and numerical data show that increased vasoconstriction is related to a higher dicrotic notch. This study is proof of concept that the dicrotic notch is mainly determined by the reflection waves and their characteristics.

We propose these experimental and numerical examples as a conceptual and educational tool to illustrate how changes in reflection waves can produce modifications in the morphology of the dicrotic notch.

\section{Conflicts of interest}

None declared.

\section{Acknowledgments}


We would like to thank the anesthesiologists and nurses from the vascular surgery ward at the Hôpital Universitaire Pitié-Salpêtrière, and also Sandra Wray, for their help and support in this study. 


\section{TABLES}

\begin{tabular}{|l|l|l|l|}
\hline $\mathrm{L}[\mathrm{mm}]$ & $\mathrm{R}[\mathrm{mm}]$ & $\mathrm{h}[\mathrm{mm}]$ & $\mathrm{E}[\mathrm{kPa}]$ \\
\hline $200-1000$ & 14.7 & 1.63 & 400 \\
\hline
\end{tabular}

Table 1. Geometrical and mechanical parameters describing the single artery network model and the parent artery of the bifurcation network model.

\begin{tabular}{|l|l|l|l|}
\hline $\mathrm{L}[\mathrm{mm}]$ & $\mathrm{R}[\mathrm{mm}]$ & $\mathrm{h}[\mathrm{mm}]$ & $\mathrm{E}[\mathrm{kPa}]$ \\
\hline 100 & 5 & 1 & 400 \\
\hline
\end{tabular}

Table 2. Geometrical and mechanical parameters describing the daughter arteries of the bifurcation network model. 


\section{FIGURES}
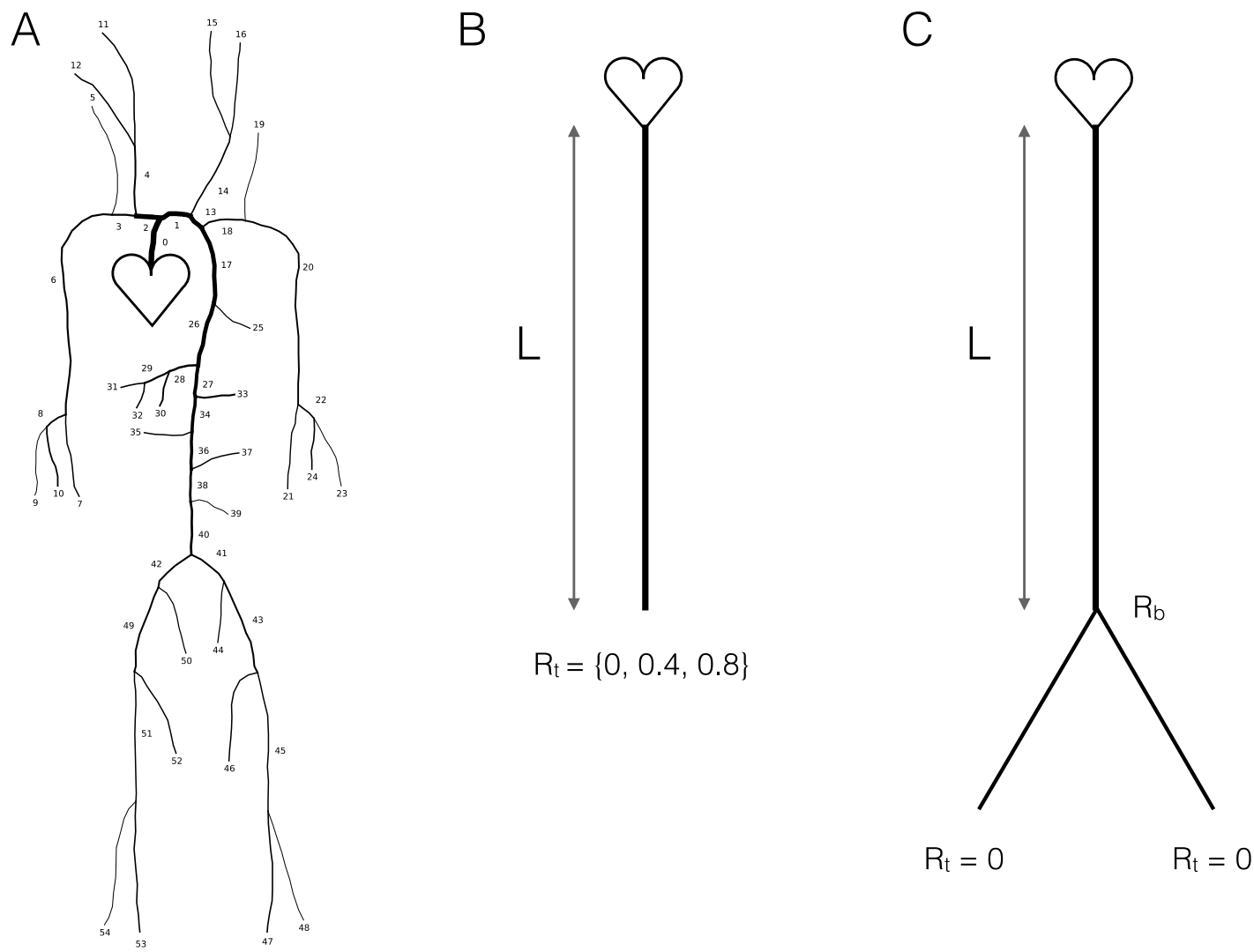

Figure 1. Three different artery models used in our simulations. A. A human arterial network of 55 segments based on vascular nuclear magnetic resonance images. The influence of the peripheral vascular bed was taken into account through terminal reflection coefficients (Rt), which could be modified and, for the sake of simplicity, had the same value for all terminal segments. B. A single segment model considering one straight artery without bifurcations, where the terminal coefficient (Rt) could be modified. The mechanical and geometrical parameters of this single segment were identical to those of the aorta of the 55 arteries model and can be found in Table 1. C. A single bifurcation model formed of one parent artery that divides into two identical daughter arteries. The geometrical and mechanical parameters of the parent artery were the same as the single artery model. The two daughter arteries were identical and their parameters are presented in Table 2. The terminal coefficient (Rt) of the daughter arteries were set to 0 in order to study the value of the reflection coefficient $(\mathrm{Rb})$ at the bifurcation, which could be modified.

\section{A. Patient 1}





\section{B. Patient 2}

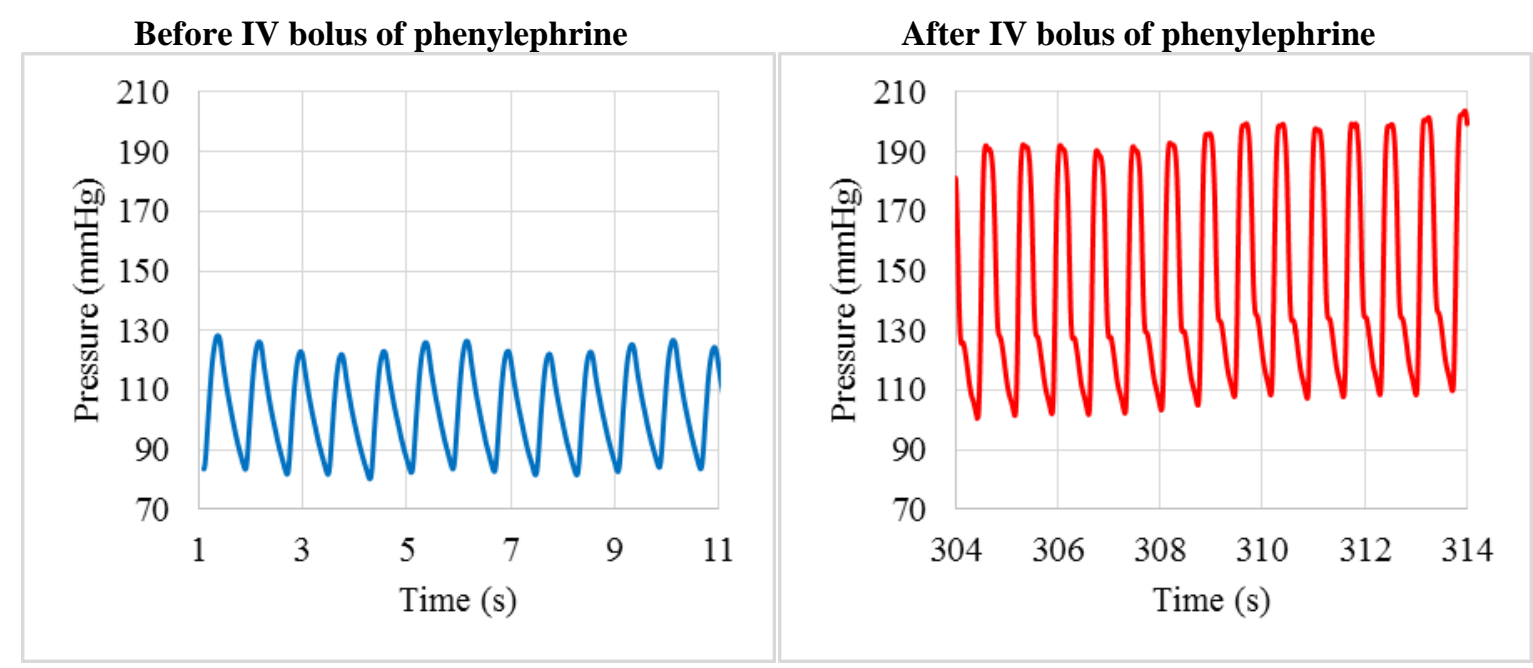

Figure 2. A. Left and middle panel: Invasive arterial pressure-time waves for Patient 1 undergoing abdominal aorta prosthetic replacement. Periodic oscillations in pressure waves were due to respiratory variations, which were enhanced due to mechanical ventilation settings. Initially the patient had a small dicrotic notch. Notice that, after an IV bolus of phenylephrine, the dicrotic notch increased its peak amplitude without changing its relative time-position within the pressure cycle. This could indicate that vasoconstriction induced by phenylephrine increases the amplitude of the dicrotic notch without modifying pressure wave travel time. Right panel: Comparison of mean pressure tracings over a 10-second interval before (continuous line) and after (dotted line) an IV bolus of phenylephrine with time normalized to the duration of one cycle and pressure normalized to the nearest inflexion point before the dicrotic notch. The proportional increase in the normalized peak value of the main wave $(2 \%)$ was smaller than the proportional increase in the normalized peak value of the dicrotic notch $(6 \%)$. B. Invasive arterial pressure-time waves for Patient 2 undergoing a right femoralpopliteal bypass. Initially the patient had a monophasic waveform that lacked any noticeable dicrotic notch. After an IV bolus of phenylephrine, blood pressure increased significantly and waveform morphology changed, with the dicrotic notch interrupting the end of the diastolic slope. 
A

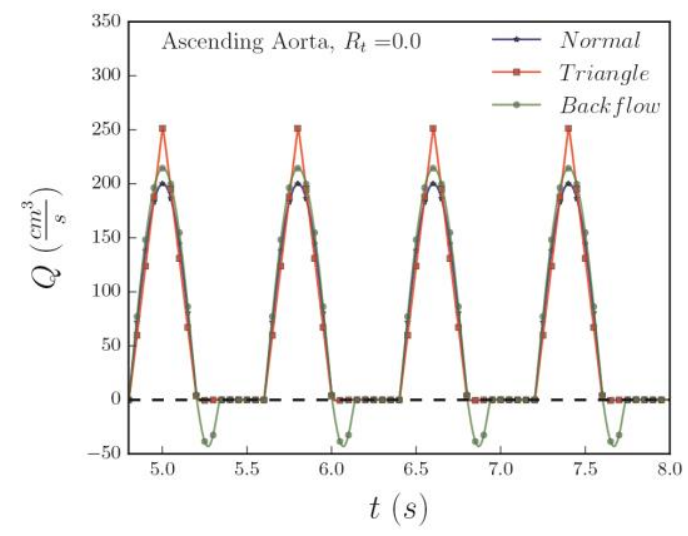

B

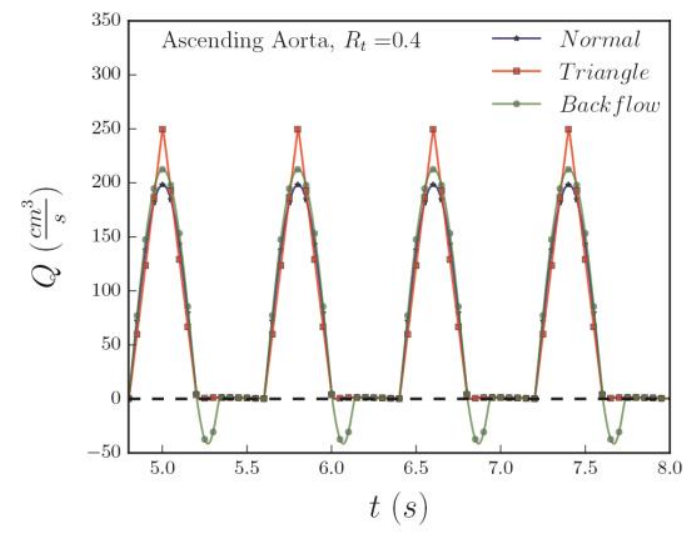

$\mathrm{C}$

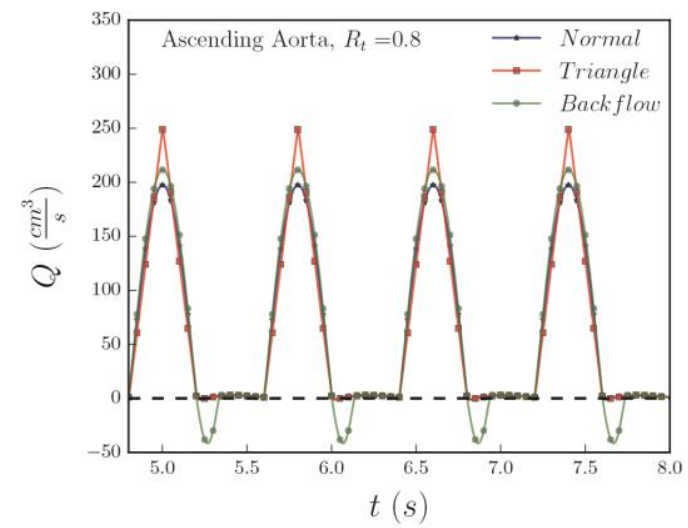

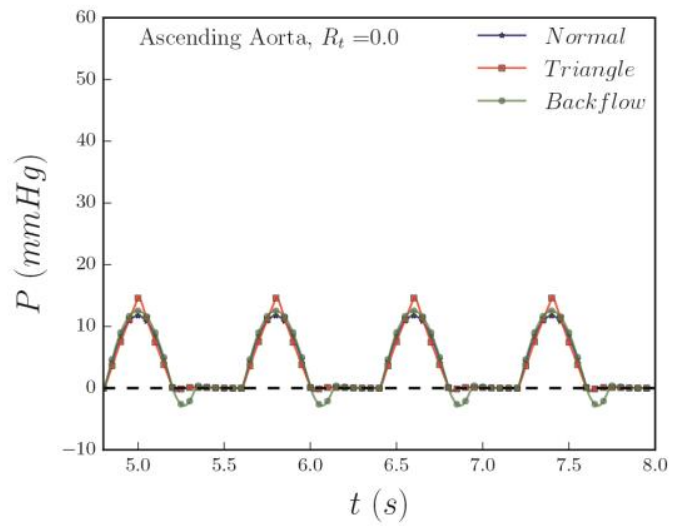
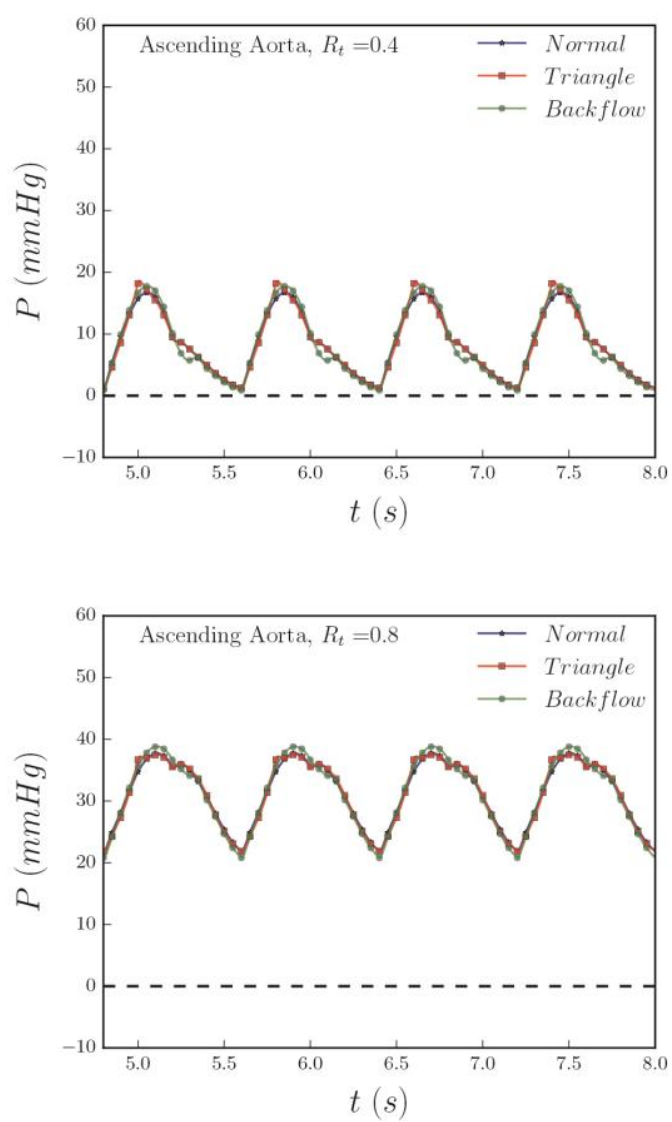

Figure 3. Numerical simulation of flow rate $(\mathrm{Q})$ and pressure $(\mathrm{P})$ time curves in the proximal aorta of the 55-segment vascular network, using different terminal reflection coefficients (Rt). A. A terminal coefficient of $\mathrm{Rt}=0$ yields a monophasic pressure-time curve that practically mirrors flow-time curves. Note that the dicrotic notch is undetectable. B. A terminal coefficient of $\mathrm{Rt}=0.4$ results in a clear dicrotic notch which interrupts the downward pressure slope of the original pressure wave. C. A high terminal reflection coefficient of $\mathrm{Rt}=0.8$ produces a higher dicrotic notch that is overimposed on the original pressure wave nearly at its peak. Three different inlet flow rate signals are illustrated: a parabolic signal without backflow (normal), a parabolic signal with backflow (blackflow), and a triangular signal without backflow (triangular). The presence of backflow, as well as the shape of the inlet signal, modifies the shape but not the time-position of the dicrotic notch. 
A

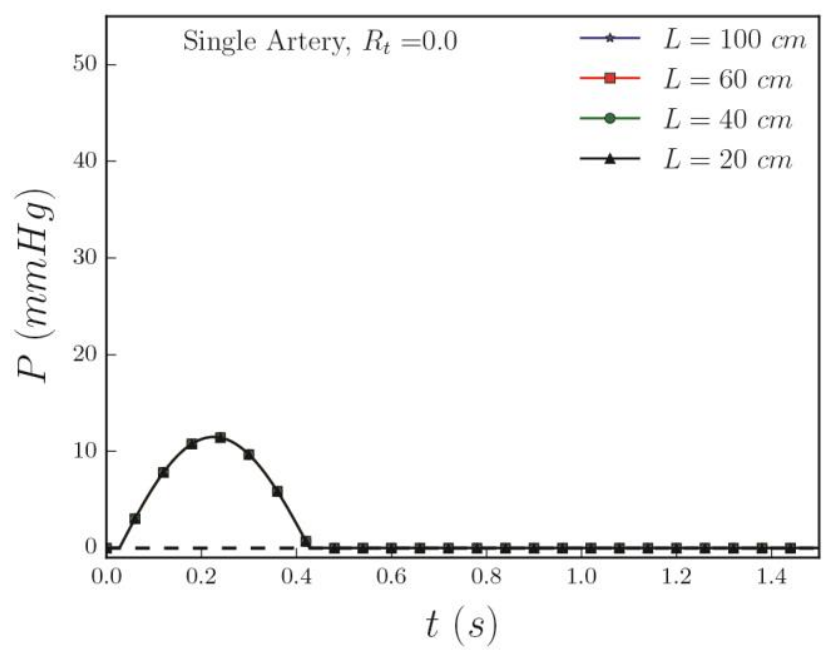

B

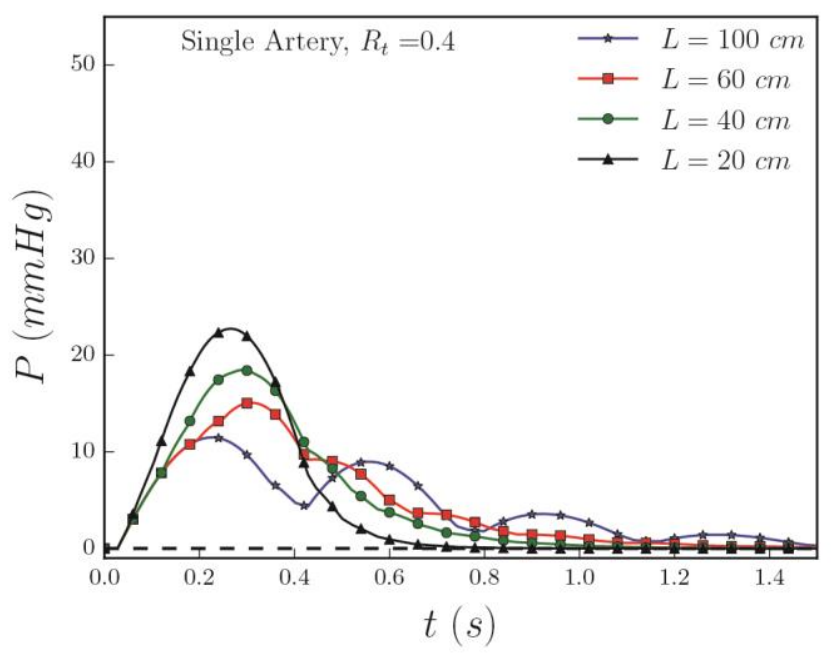

$\mathrm{C}$

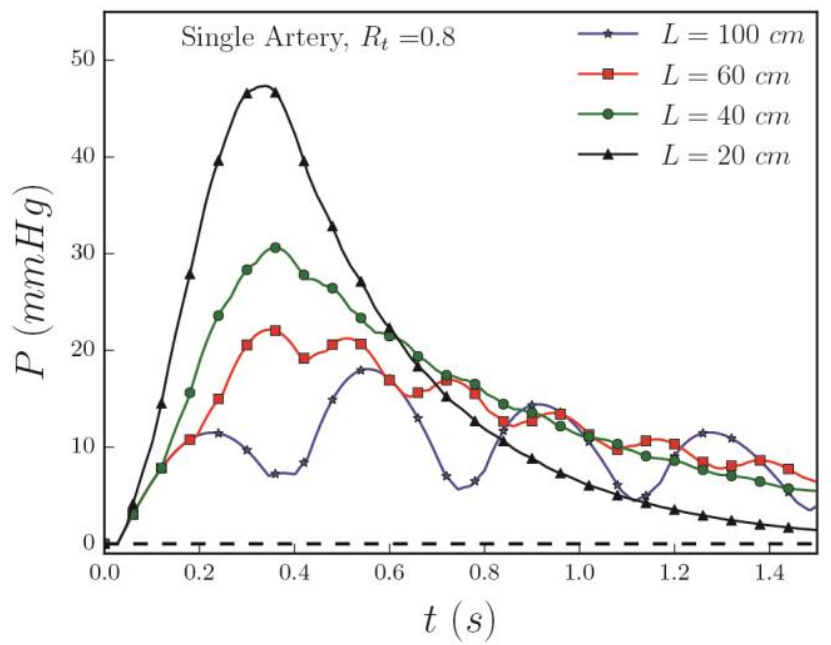

Figure 4. Pressure-time curve of a single beat impulse in a one-artery model without bifurcations, measured at $150 \mathrm{~mm}$ from the beginning of the artery, in arteries of different lengths. These simulations considered a pulse period of 0.1 seconds and only pure wave propagation. A. Considering a reflection coefficient of $\mathrm{Rt}=0$, our simulation shows a single original pressure wave that is similar regardless of the vessel length, and no reflected waves at all. B. At an $\mathrm{Rt}=0.4$, our simulation shows two reflected waves that are much closer to the original pressure wave in shorter vessels than in longer 
vessels (e.g., compare the reflected waves from the $600 \mathrm{~mm}$ vessel and the $1,000 \mathrm{~mm}$ vessel). Eventually, at very short vessel lengths, the two reflection waves merge together with the original wave by forming a notch (i.e., the $200 \mathrm{~mm}$ vessel). C. At an $\mathrm{Rt}=0.8$ our simulation shows that by increasing the reflection coefficient, only the amplitudes of the waves change. The reflected waves do not get closer to one another or to the original pressure wave, indicating that they do not change their propagation velocity. 
A

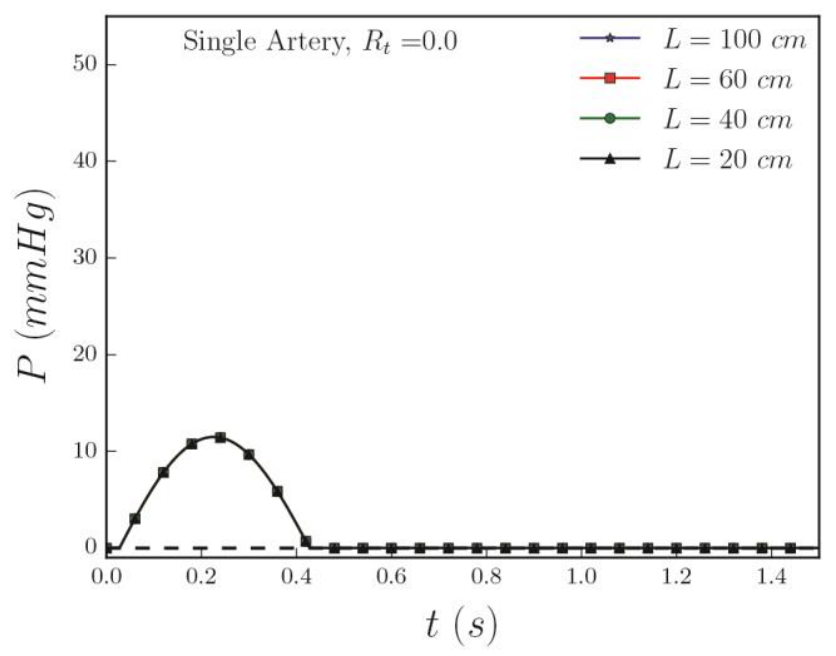

B

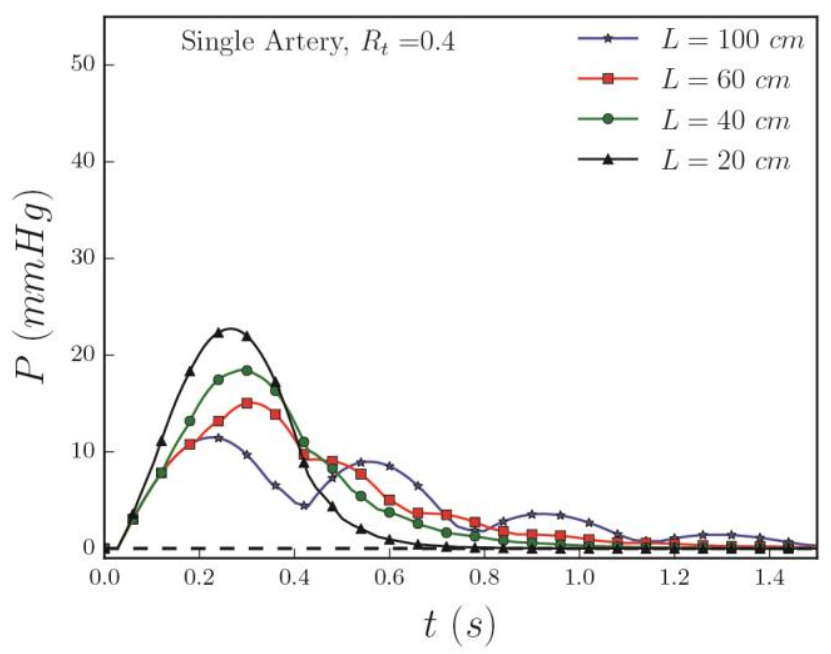

$\mathrm{C}$

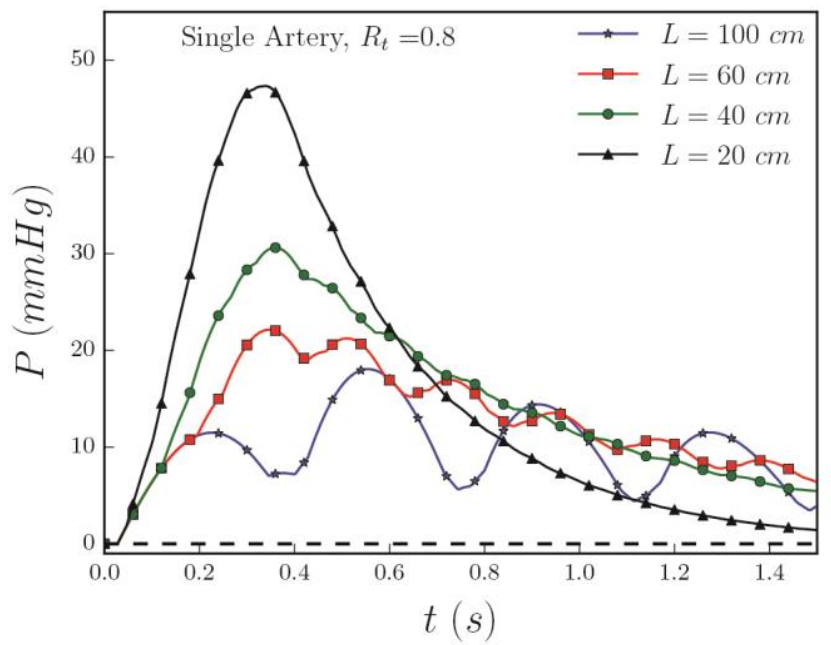

Figure 5. Pressure-time curve of a single beat impulse in a one-artery model without bifurcations measured at $150 \mathrm{~mm}$ from the beginning of the artery, in arteries of different lengths. These simulations considered a pulse period of 0.8 seconds and only pure wave propagation. A. At an $\mathrm{Rt}=0$, once again our simulation shows a single original pressure wave that is similar regardless of the vessel length, and no reflected waves at all. B. At an $\mathrm{Rt}=0.4$ and a longer pulse period than in Figure 4, our simulation shows two reflected waves that merge with the original pressure wave even in vessels 
with long lengths (e.g., in the $600 \mathrm{~mm}$ vessel they are almost completely merged together). C. At an Rt=0.8. By increasing the reflection coefficient, the amplitudes of the waves change. With a longer pulse period, the individual elements of these pressure waves are harder to point out. 
A

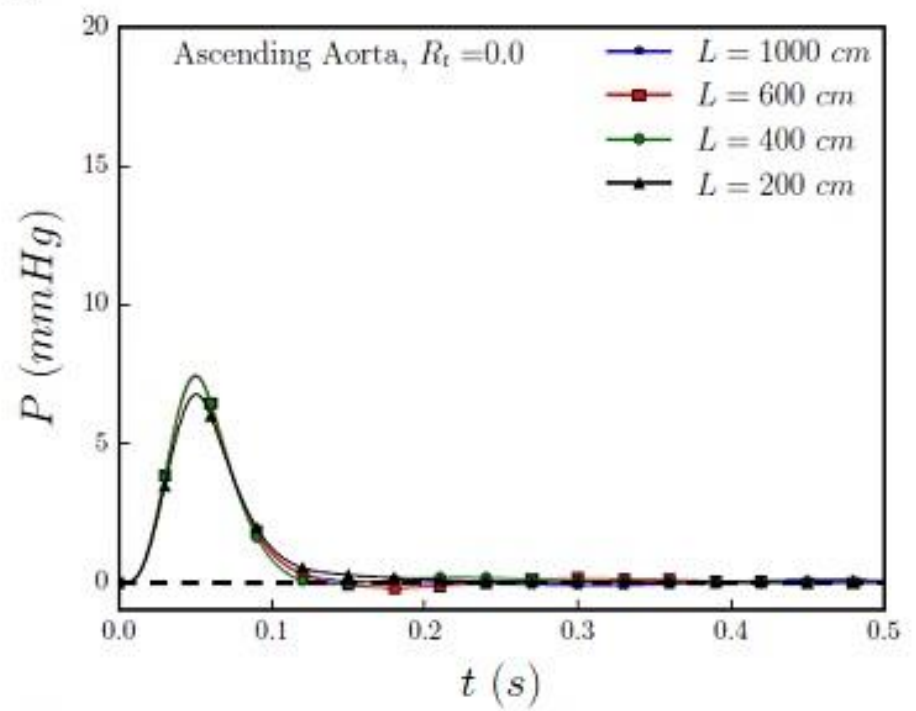

B

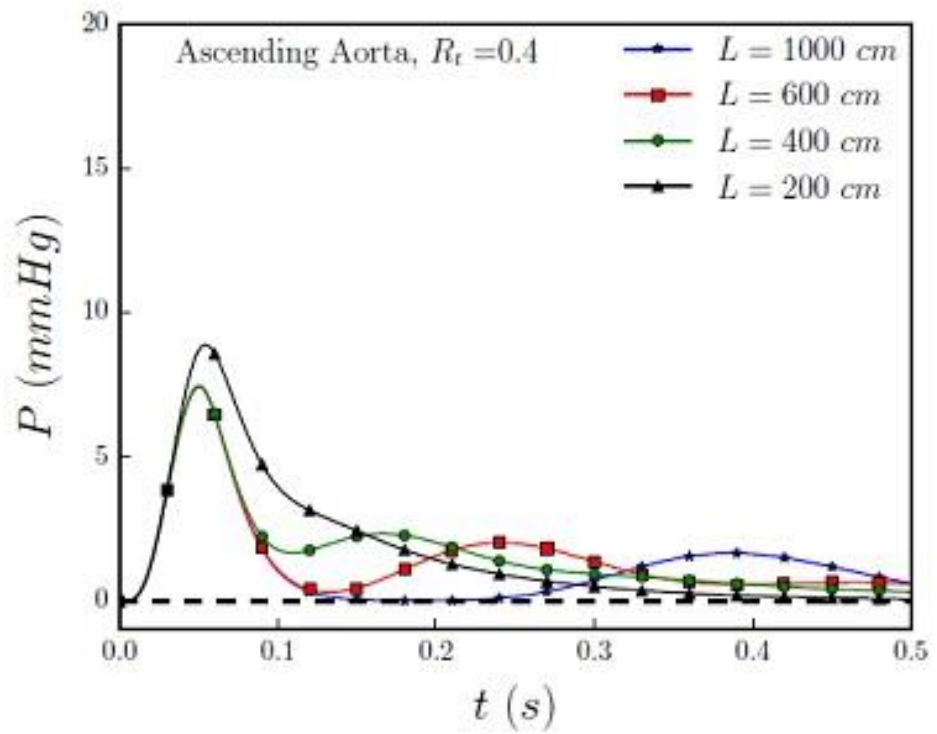

C

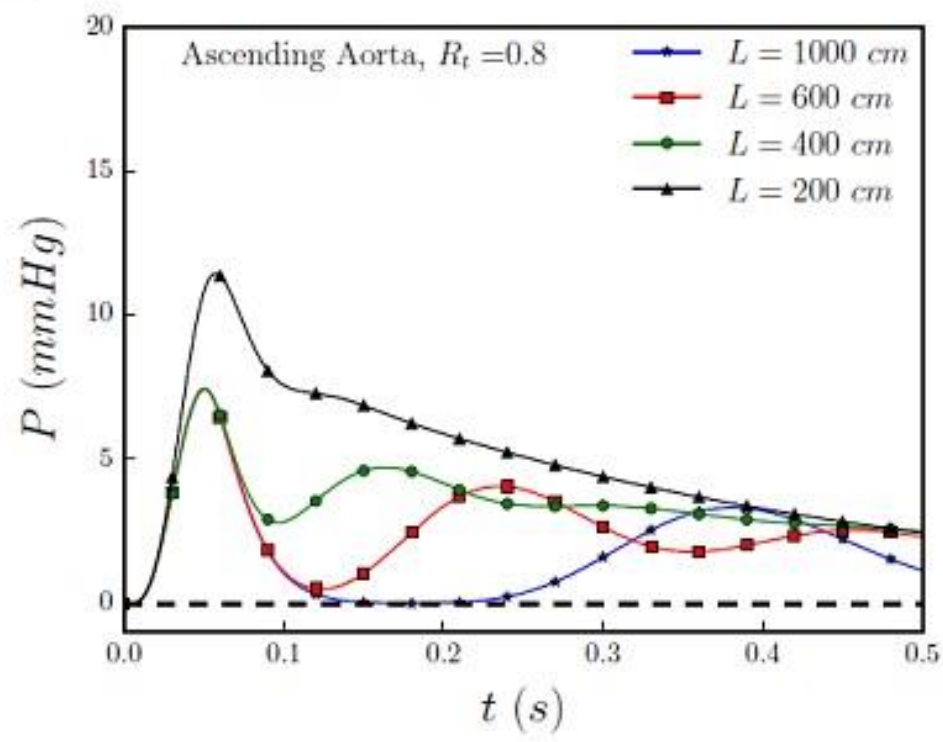


A

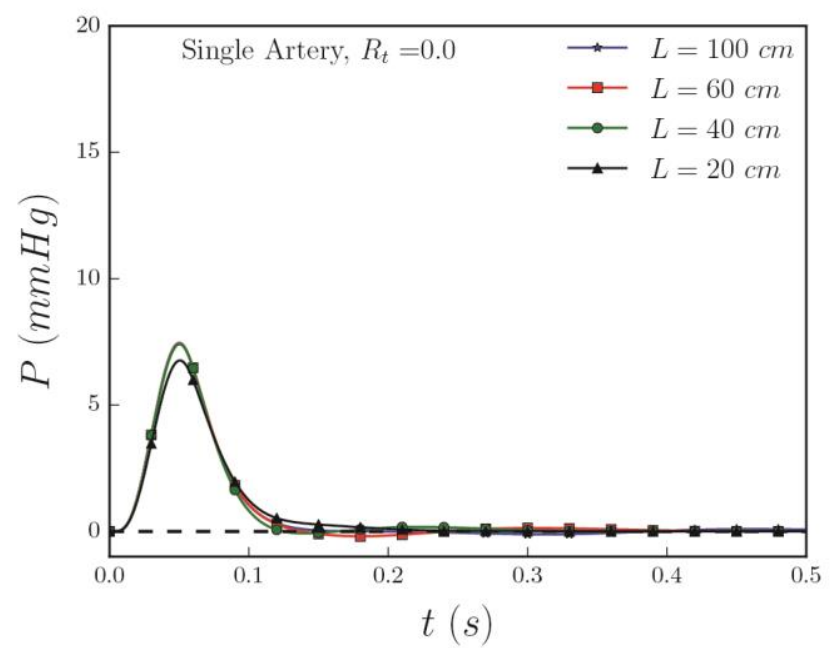

B

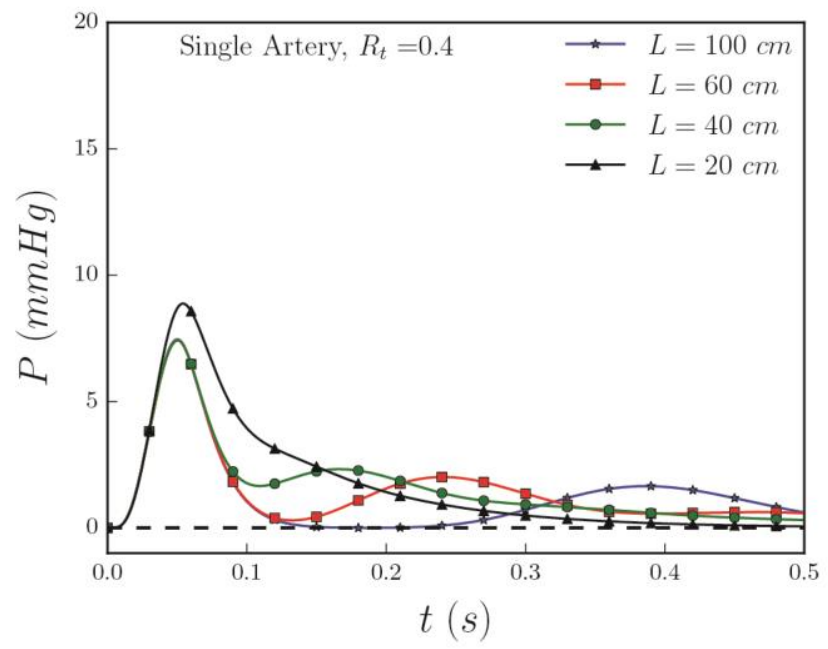

$\mathrm{C}$



Figure 6. Pressure-time curve of a single beat impulse in a one-artery model without bifurcations measured at $150 \mathrm{~mm}$ from the beginning of the artery, in arteries of different lengths. These simulations considered a pulse period of 0.1 seconds and the influence of viscous and viscoelastic effects on wave propagation. A. At an $\mathrm{Rt}=0$ our simulation shows a single original pressure wave that is similar regardless of the vessel length, and no reflected waves. B. At an $\mathrm{Rt}=0.4$. In comparison to pure wave propagation, reflected waves merge with the original pressure wave at longer lengths 
(e.g., in the $400 \mathrm{~mm}$ vessel). C. At an $\mathrm{Rt}=0.8$ the compliant effect of viscoelasticity "smoothens the curves out", resulting in the overlap of waves that previously were identified as individual elements when considering only pure wave propagation. 
A

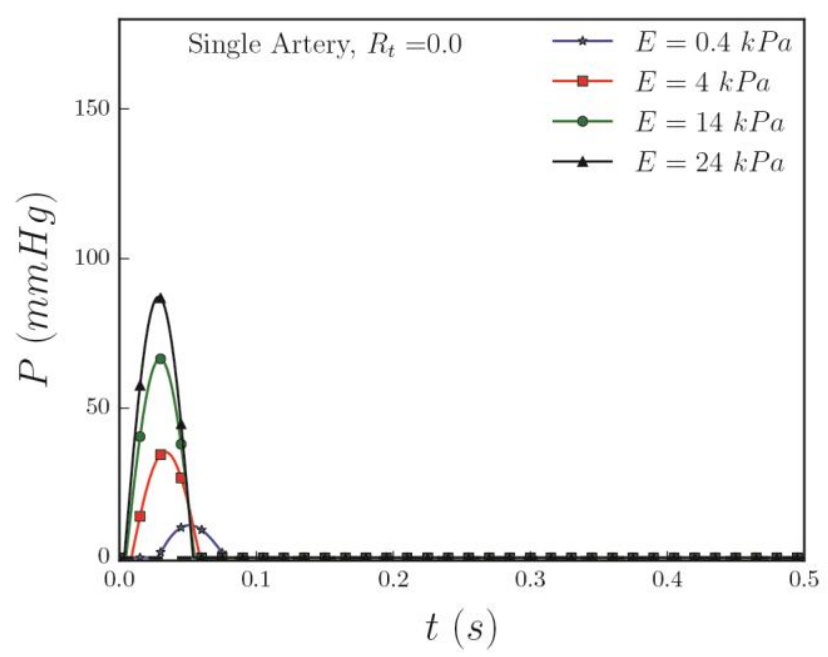

B

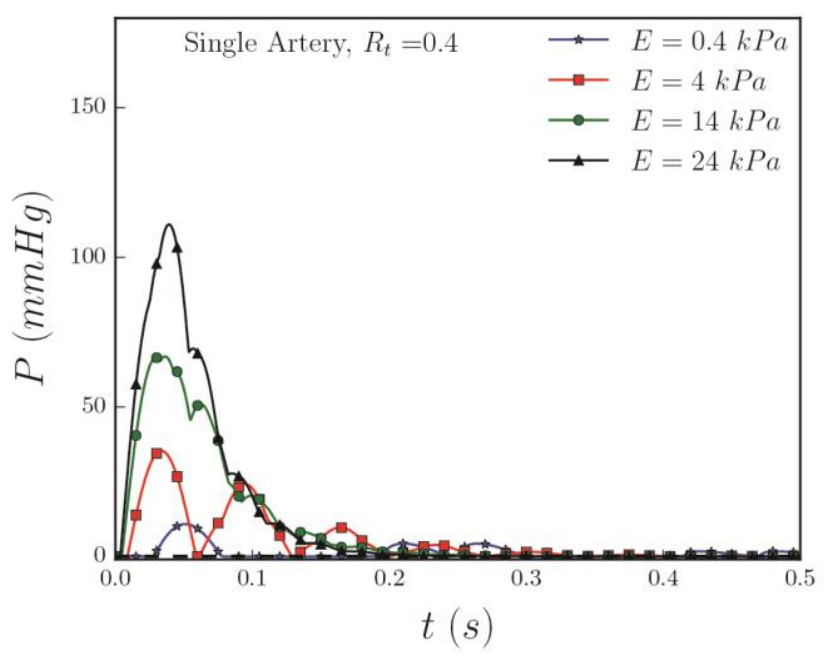

$\mathrm{C}$

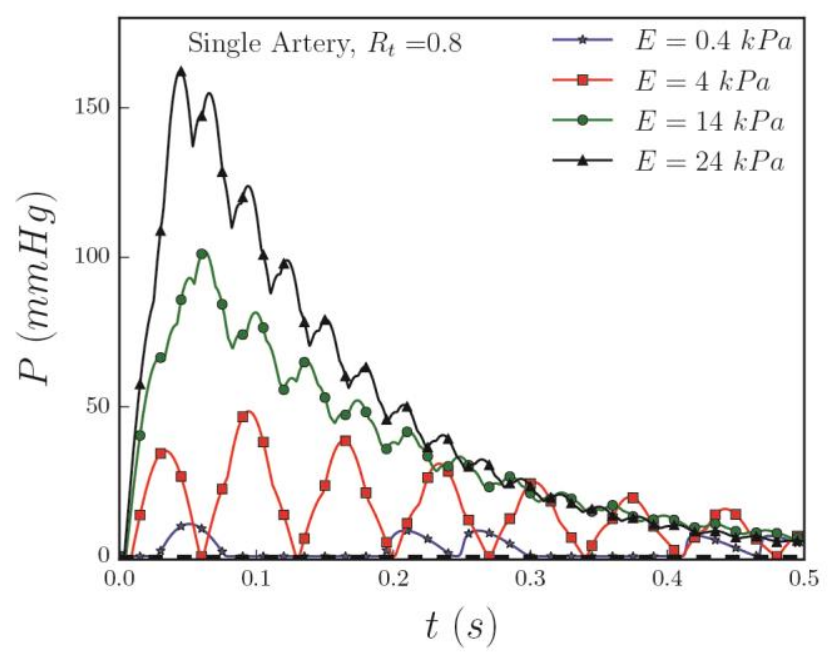

Figure 7. Pressure $(\mathrm{P})$ time curves of a single beat impulse in a one-artery model without bifurcations measured at $150 \mathrm{~mm}$ from the beginning of an artery with a length of $600 \mathrm{~mm}$. These simulations considered a pulse period of 0.1 seconds and viscous and viscoelastic effects on wave propagation. A. Considering a reflection coefficient (Rt) of 0 -therefore, in the absence of reflection waves- increases in vessel elasticity (E) raised the propagation speed but did not create a dicrotic notch. B. Considering a reflection 
coefficient (Rt) of 0.4, reflection waves appear. An increase in the vessel's elasticity (E) increased the amplitude and the propagation speed of both the original pressure wave and the reflected wave, which therefore appear earlier. C. Considering a reflection coefficient (Rt) of 0.8 , reflection waves increased their amplitude and reduced their timing. 




Figure 8. Pressure-time curve of a single beat impulse in an artery model with bifurcations, measured at $150 \mathrm{~mm}$ from the beginning of the parent artery, in arteries of different lengths. These simulations considered a pulse period of 0.1 seconds, a reflection coefficient (Rt) of 0.4 and only pure wave propagation. Our simulation is identical to that obtained for the single-artery model without bifurcations (Figure 4B) and demonstrates that internal reflection can also play an important role and influence the shape of the dicrotic notch. 


\section{References}

1. Alastruey J, Parker KH, Peiró J, Sherwin SJ. Analyzing the pattern of pulse waves in arterial networks: a time-domain study. Journal of Engineering Mathematics. 1;64(4):331-51, 2009.

2. Alastruey J, Khir AW, Matthys KS, Segers P, Sherwin SJ, Verdonck PR, Parker KH, Peiró J. Pulse wave propagation in a model human arterial network: assessment of 1-D visco-elastic simulations against in vitro measurements. Journal of Biomechanics. 44(12):2250-2258, 2011.

3. Ambrosi D, Quarteroni A, Rozza G. Modeling of physiological flows. Vol. 5. Springer Science \& Business Media, 2012.

4. Borlotti A, Li Y, Parker KH, Khir AW. Experimental evaluation of local wave speed in the presence of reflected waves. Journal of Biomechanics. 47(1):87-95, 2004.

5. Boulpaep EL, Boron WF, Caplan MJ, Cantley L, Igarashi P, Aronson PS, Moczydlowski EG. Medical Physiology a Cellular and Molecular Approach. Saunders, 2009. Chapter 22, The Heart as a Pump, p798.

6. Caro CG, Pedley TJ, Schroter RC, Seed WA. The mechanics of the circulation. Cambridge University Press, 2012.

7. Chemla D, Hebert JL, Coirault C, Salmeron S, Zamani K, Lecarpentier Y. Matching dicrotic notch and mean pulmonary artery pressures: implications for effective arterial elastance. American Journal of Physiology-Heart and Circulatory Physiology. 271 (4): 1287-1295, 1996.

8. Dahlgren G, Veintemilla F, Settergren G, Liska J. Left ventricular end-systolic pressure estimated from measurements in a peripheral artery. J Cardiothorac Vasc Anesth. 5(6):551-3, 1991.

9. Falsetti, H.L, Mates RE, Carroll RJ, Gupta RL, Bell AC. Analysis and correction of pressure wave distortion in fluid-filled catheter systems. Circulation. 1974: 49(1):165-172.

10. Guyton AC, Hall JE. Textbook of Medical Physiology. 11th edition. Elsevier Inc, 2006. Philadelphia PA. Chapter 9, Heart Muscle; The Heart as a Pump and Function of the Heart Valves, p109.

11. Hebert JL, Lecarpentier Y, Zamani K, Coirault C, Daccache G, Chemla D, Wuilliez N, Larsonneur L. Relation between aortic dicrotic notch pressure and mean aortic pressure in adults. The American Journal of Cardiology. 76 (4): 301-306, 1995.

12. Hermeling E, Reesink KD, Kornmann LM, Reneman RS, Hoeks AP. The dicrotic notch as alternative time-reference point to measure local pulse wave velocity in the carotid artery by means of ultrasonography. J Hypertens. 27:2028 -2035, 2009.

13. Hermeling E, Hoeks AP, Winkens MH, Waltenberger JL, Reneman RS, Kroon AA, Reesink KD. Noninvasive assessment of arterial stiffness should discriminate between systolic and diastolic pressure ranges. Hypertension. 55(1):124-30, 2010.

14. Higashidate M, Tamiya K, Beppu T, Imai Y. Regulation of the aortic valve opening: in vivo dynamic measurement of aortic valve orifice area. The Journal of Thoracic and Cardiovascular Surgery. 110(2): 496-503, 1995.

15. Hirschfeld S, Liebman NJ, Borkat G, Bormuth C. Intracardiac pressure-sound correlates of echographic aortic valve closure. Circulation. 55(4), 602-604, 1977.

16. Khir AW, Parker KH. Measurements of wave speed and reflected waves in elastic tubes and bifurcations. Journal of Biomechanics. 35(6):775-783, 2002.

17. Latham RD, Westerhof N, Sipkema P, Rubal BJ, Reuderink P, Murgo JP. Regional wave travel and reflections along the human aorta: a study with six simultaneous micromanometric pressures.Circulation, 1985, 72(6), 1257-1269.

18. Levy MN, Berne RM, Koeppen BM, Stanton BA. Berne \& Levy Principles of Physiology, $5^{\text {th }}$ Edition. Mosby, 2006. Chapter 15, Hemodynamics. Relationship between pressure and flow.

19. Mann, DL, Zipes DP, Libby P, Bonow RO. Braunwald's heart disease: a textbook of cardiovascular medicine. Elsevier Health Sciences, 2014. Chater 3, Evaluation of the patient, p100.

20. Marino PL. Chapter 7: Arterial pressure monitoring. In: Marino's the ICU Book. Lippincott Williams \& Wilkins, 2014. p 135.

21. Matthys KS, Alastruey J, Peiró J, Khir AW, Segers P, Verdonck PR, Parker KH, Sherwin SJ. Pulse wave propagation in a model human arterial network: Assessment of 1-D numerical simulations against in vitro measurements. Journal of Biomechanics. 40(15);3476-3486, 2007.

22. Murgo JP, Westerhof NI, Giolma JP, Altobelli SA. Aortic input impedance in normal man: relationship to pressure wave forms. Circulation. 62(1):105-16, 1980.

23. Mynard JP, Nithiarasu P. A 1D arterial blood flow model incorporating ventricular pressure, aortic valve and regional coronary flow using the locally conservative Galerkin (LCG) method. Commun. Numer. Meth. Engng. 24:367-417, 2008

24. O'Rourke MF, Avolio AP. Pulsatile flow and pressure in human systemic arteries. Studies in man and in a multibranched model of the human systemic arterial tree. Circ. Res. 46, 363-372, 1980.

25. O'Rourke MF, Staessen JA, Vlachopoulos C, Duprez D, Plante GE. Clinical applications of arterial stiffness: definitions and reference values. Am J Hypertens. 15:426-444, 2002.

26. Pinsky MR, Brochard L, Mancebo J, Pinsky MR. Applied physiology in intensive care medicine. Berlin: Springer. 2006. P 90.

27. Segers P, Stergiopulos N, Verdonck P, Verhoeven R. Assessment of distributed arterial network models. Medical and Biological Engineering and Computing. 35(6):729-736, 1997.

28. Shinozaki T, Deane RS, Mazuzan JE. The dynamic responses of liquid-filled catheter systems for direct measurements of blood pressure. Anesthesiology. 1980 Dec; 53(6):498-504

29. UpToDate. Waveforms by location of the pulmonary artery (PA) catheter tip (Accessed August 13 ${ }^{\text {th }}$, 2015): http://www.uptodate.com/contents/search?search=dicrotic+notch\&sp=0\&searchType=GRAPHICS\&source=USER_INPUT\&s earchControl=TOP_PULLDOWN\&searchOffset=

30. Vanden Bos GC, Westerhof N, Randall OS: Pulse wave reflection: can it explain the differences between systemic and pulmonary pressure and flow waves? Circ Res 51: 479, 1982.

31. Wang X, Fullana JM, Lagrée PY. Verification and comparison of four numerical schemes for a 1D viscoelastic blood flow model. Computer methods in biomechanics and biomedical engineering. 18(15),1704-1725, 2015.

32. Westerhof N, Sipkema P, Vanden Bos GC, Elzinga G: Forward and backward waves in the arterial system. Cardiovasc Res 6: $648,1972$.

33. Westerhof B.E., et al. Quantification of wave reflection in the human aorta from pressure alone: a proof of principle. Hypertension. 48: 595-601, 2006.

34. Zundel MT, Pattyn M, Chelimsky TC, Riess ML. Arterial flow waveforms, vascular tone, and chronic fatigue: a case report. Auton Neurosci. 190:58-60, 2015. 